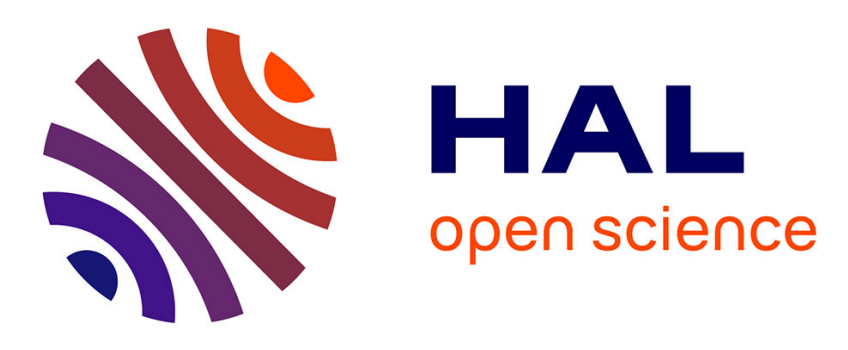

\title{
EMC Modeling of an Industrial Variable Speed Drive With an Adapted PEEC Method
}

Vincent Ardon, Jérémie Aimé, Olivier Chadebec, Edith Clavel, Jean-Michel Guichon, Enrico Vialardi

\section{- To cite this version:}

Vincent Ardon, Jérémie Aimé, Olivier Chadebec, Edith Clavel, Jean-Michel Guichon, et al.. EMC Modeling of an Industrial Variable Speed Drive With an Adapted PEEC Method. IEEE Transactions on Magnetics, 2010, 46 (8), pp 2892-2898. 10.1109/TMAG.2010.2043420 . hal-00521938

\section{HAL Id: hal-00521938 \\ https://hal.science/hal-00521938}

Submitted on 29 Sep 2010

HAL is a multi-disciplinary open access archive for the deposit and dissemination of scientific research documents, whether they are published or not. The documents may come from teaching and research institutions in France or abroad, or from public or private research centers.
L'archive ouverte pluridisciplinaire HAL, est destinée au dépôt et à la diffusion de documents scientifiques de niveau recherche, publiés ou non, émanant des établissements d'enseignement et de recherche français ou étrangers, des laboratoires publics ou privés. 


\title{
EMC Modeling of an Industrial Variable Speed Drive With an Adapted PEEC Method
}

\author{
Vincent Ardon ${ }^{1,2}$, Jérémie Aime ${ }^{1,3}$, Olivier Chadebec ${ }^{1}$, Edith Clavel ${ }^{1}$, Jean-Michel Guichon ${ }^{1}$, and \\ Enrico Vialardi ${ }^{1}$ \\ ${ }^{1}$ Grenoble Electrical Engineering Laboratory, Université de Grenoble (Grenoble-INP, UJF, UMR CNRS 5269), \\ 38402 Grenoble, France \\ ${ }^{2}$ Technological Department-Software, Cedrat, 38246 Meylan, France \\ ${ }^{3}$ Schneider and Toshiba Inverter Europe, 27120 Pacy sur Eure, France
}

\begin{abstract}
This paper presents an adapted partial element equivalent circuit (PEEC)-based methodology applied to the modeling of interconnections of power electronics devices. Although this method is already well known, the originality of this work is its use to model a device presenting an industrial complexity. To make possible this modeling, two adapted integral methods, based on two different meshings, are presented. They are dedicated respectively to the computation of parasitic inductances and capacitances and lead to an equivalent circuit of the system. From a time-domain simulation of this circuit, current and voltage sources can be extracted and used to compute the radiated near magnetic field. This approach has been applied to model a real industrial static converter via system couplings, a variable speed drive. Good agreements have been obtained between simulated and measured results on conducted and emitted electromagnetic analysis.
\end{abstract}

Index Terms-Electromagnetic compatibility, fast multipole method, parasitic capacitances, parasitic elements, partial element equivalent circuit (PEEC), power electronics, power interconnections.

\section{INTRODUCTION}

$\mathbf{N}$ OWADAYS, power-electronic designers need more and more accurate modeling tools able to simulate complex geometries, in order to save money and time on tests. Recent works and researches trends [1] have proven that this can be achieved by means of a system coupling approach and the use of a complete electrical equivalent circuit of the device. This circuit approach is interesting because it avoids iterations between different modeling software: in the equivalent circuit, physical and electrical behaviors are modeled together. For example, to analyze the electromagnetic compatibility (EMC) performances with respect to standards which are becoming more and more stringent, it is necessary to model common and differential mode currents flowing into the power interconnections, and consequently, common and differential mode loops-via parasitic capacitances - appear and emit magnetic field into the device. The constraints within power-electronics structures are the following.

- The frequency range is comprised between $10 \mathrm{kHz}$ and 100 or $200 \mathrm{MHz}$ - so the propagation effects can be neglected because the typical dimension of devices is about some tens of centimeters. The wavelength at $200 \mathrm{MHz}$ is $\lambda=1.5 \mathrm{~m}$ and the longest conductor $\mathrm{L}$ is around $15 \mathrm{~cm}(\mathrm{~L}<\lambda / 10)$. On the other hand, the skin effects have to be taken into account because the conductors have a width of tens of millimeters. Besides the capacitive effects can not be neglected because they generally appear from around $1 \mathrm{MHz}$ (depending on geometric dimensions of conductors) and are needed in the modeling of common mode current loops that develop between the tracks and the ground planes.

Manuscript received December 22, 2009; accepted February 07, 2010. Current version published July 21, 2010. Corresponding author: V. Ardon (e-mail: vincent.ardon@g2elab.grenoble-inp.fr).

Color versions of one or more of the figures in this paper are available online at http://ieeexplore.ieee.org.

Digital Object Identifier 10.1109/TMAG.2010.2043420
- The space occupied by air medium is abundant and greater than the volume of conductors.

- Geometries are often complex, compact, irregular, and composed by multilayers of conductors separated by dielectric substrates.

The problem can be better formalized by considering the integral form of the Maxwell's equations and by assuming:

- quasi-static conditions;

- only surface location for the free-charges $\rho$;

- currents I are assumed uniform and constant in each volume element of conductors,

— vacuum permeability $\mu_{0}$ surrounding the objects;

- a homogeneous medium of permittivity $\varepsilon=\varepsilon_{\mathrm{r}} \cdot \varepsilon_{0}$;

- neglected losses in dielectric materials.

In such conditions, the external applied electric field $\mathbf{E}$ applied at a point $\mathbf{r}$ of a conductor at the pulsation $\omega$ can be written as (1) where $\mathbf{J}$ is current density, $\sigma$ the material conductivity, A the magnetic vector potential, $\varphi$ the electric potential and $G$ $\left(\mathbf{r}, \mathbf{r}^{\prime}\right)=1 /\left(4 \pi\left\|\mathbf{r}-\mathbf{r}^{\prime}\right\|\right)$ the Green function [1]

$$
\begin{aligned}
\mathbf{E}(\mathbf{r})= & \frac{\mathbf{J}(\mathbf{r})}{\sigma}+\mathrm{j} \omega \mathbf{A}(\mathbf{r})+\nabla \phi(\mathbf{r}) \\
= & \frac{\mathbf{J}(\mathbf{r})}{\sigma}+\mathrm{j} \omega \mu_{0} \iint_{\mathrm{V}} \mathrm{G}\left(\mathbf{r}, \mathbf{r}^{\prime}\right) \mathbf{J}\left(\mathbf{r}^{\prime}\right) \mathrm{dV} \\
& +\frac{1}{\varepsilon} \nabla \iint_{\mathrm{S}} \mathrm{G}\left(\mathbf{r}, \mathbf{r}^{\prime}\right) \rho\left(\mathrm{r}^{\prime}\right) \mathrm{d} \mathrm{S} .
\end{aligned}
$$

The three parts of (1) are respectively due to resistive, inductive and capacitive behaviors, which represent the main effects that have to be taken into account in the power-interconnection modeling.

To model a complex industrial device, the finite element method [2] would be little adapted regarding the difficulty to properly mesh volume air regions and conductors to accurately take into account skin and proximity effects. The use of approximated analytical formulas or multiconductor transmission 
line theory [3] is also to banish because they are not accurate enough and the medium is not always homogeneous. On the contrary, the partial element equivalent circuit (PEEC) method (i.e., extraction of equivalent circuit components thanks to integral approach) is known to be well adapted to model complex geometries with an important surrounded air region [1]-[4]. However, the classical PEEC method which is widely used at high frequencies (antennas, RF devices, etc.), would be very memory-consuming and not adapted to the consideration of skin effects, as it is based on dual inductive and capacitive meshings. This method would require the computation of a parasitic capacitance between each surface mesh element of conductors, and to take into account accurately the skin effects, the meshing would need to be refined on the conductor sides (an adapted meshing with two mesh elements in the skin depth). Consequently, the inductive effects would be difficult to model and on the contrary, capacitive effects too detailed depending on the frequency range.

In this paper, an adapted technique that takes advantages from different integral methods to analyze the performances of an industrial variable speed drive is proposed. The modeling strategy is based on the extraction of two series of equivalent electrical parameters and on a scheme for coupling them into a global circuit. In fact, on the one hand, parasitic resistances (R), inductances and mutual-inductances (L-M) are computed from a volume meshing of the conductors. On the other hand, capacitances (C) are extracted from free-charges located on conductor and dielectric interfaces. A non-necessarily conformal surface meshing adapted to the spatial location of free-charges is used in order to limit the number of surface elements and to improve the modeling of side effects.

Those extractions of equivalent parasitic components are presented in the two next sections, whereas in Section IV, the construction of the complete equivalent circuit is detailed. In the last section, the proposed approach is tested by modeling an industrial variable speed drive and some comparisons between simulations and measurements are carried out: the behavior of the harmonic response and the emitted near magnetic field are analyzed and compared to measurements.

\section{EXtraction of Parasitic Equivalent Resistances AND INDUCTANCES}

This section details methods and meshing techniques used for the computation of parasitic elements which model the resistive and the inductive behaviors of an interconnection structure. The volume of the conductor is meshed into parallelepiped elements where the current density is assumed uniform. The main advantage of our approach is the fact that the meshing depends on a chosen frequency. To properly model skin and proximity effects, conductors must be considered either unidirectional (thin or long tracks) or bidirectional (large tracks and ground planes), depending on the directions of the flowing current. The conductors belonging to the first type are meshed in the skin depth (at least two elements in the skin depth) but not in the length (gain in number of elements), whereas the bidirectional ones are discretized in two directions.

To compute a parasitic resistance $\mathrm{R}_{\mathrm{i}}$ in each volume element $\mathrm{V}_{\mathrm{i}}$ of length $\mathrm{l}_{\mathrm{i}}$, section $\mathrm{S}_{\mathrm{i}}$, and resistivity $\rho$, the following analytical formula is used:

$$
\mathrm{R}_{\mathrm{i}}=\rho \frac{\mathrm{l}_{\mathrm{i}}}{\mathrm{S}_{\mathrm{i}}}
$$

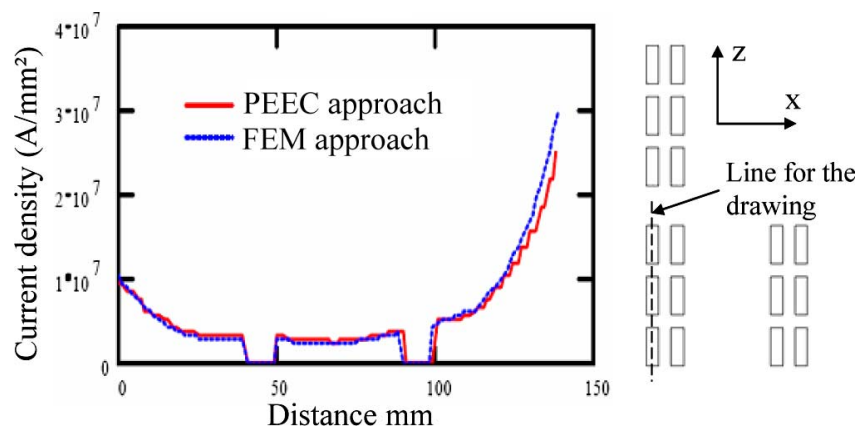

Fig. 1. Three-phase busbar system (six bars by phase).

Each mesh element $V_{i}$ presents also a self inductance $L_{i}$ depending from its section $S_{i}$ and its length $l_{i}(3)$. Moreover, each couple of nonperpendicular elements is also characterized by a mutual inductance which is computed by integrating the magnetic vector potential created by one volume element $V_{i}$ on the other $V_{\mathbf{j}}$ and with the unit vectors $\mathbf{u}_{\mathbf{i}}$ and $\mathbf{u}_{\mathbf{j}}$ which define the direction of the flowing current

$$
\left\{\begin{array}{l}
\mathrm{L}_{\mathrm{i}}=\frac{\mu_{0}}{\mathrm{~S}_{\mathrm{i}}} \iiint \int_{\mathrm{V}_{\mathrm{i}}}\left(\iiint_{\mathrm{V}_{\mathrm{i}}} \mathrm{G}\left(\mathrm{r}_{\mathrm{i}}, \mathrm{r}_{\mathrm{i}}^{\prime}\right) \mathrm{dV}_{\mathrm{i}}\right) \mathrm{d} \mathrm{V}_{\mathrm{i}}^{\prime} \\
\mathrm{M}_{\mathrm{ij}}=\mu_{0} \frac{\mathrm{u}_{\mathrm{i}} \cdot \mathrm{u}_{\mathrm{j}}}{\mathrm{S}_{\mathrm{i}} \mathrm{S}_{\mathrm{j}}} \iiint_{\mathrm{V}_{\mathrm{j}}}\left(\iiint_{\mathrm{V}_{\mathrm{i}}} \mathrm{G}\left(\mathrm{r}_{\mathrm{i}}, \mathrm{r}_{\mathrm{j}}\right) \mathrm{d} \mathrm{V}_{\mathrm{i}}\right) \mathrm{d} \mathrm{V}_{\mathrm{j}}
\end{array} .\right.
$$

Because of the parallelepiped shape of the elements the double integral in $\mathrm{L}_{\mathrm{i}}$ can be expressed in an analytical form and easily computed. The mutual inductances $\mathrm{M}_{\mathrm{ij}}$ are computed thanks to a analytical/numerical integration technique (an analytical expression for the first integral is used, the second one being computed thanks to an adaptive gauss point integration ensuring a good accuracy). It is worth to notice that the mesh elements are not necessarily parallel. All values of $\mathrm{L}_{\mathrm{i}}$ and $\mathrm{M}_{\mathrm{ij}}$ can then be organized in a dense and square matrix [L-M] whose size is equal to the number of mesh elements [5].

To illustrate our approach let us consider a 3-phase busbar system, where each phase is constituted by six parallel bars (Fig. 1, on the right) [6]. The distribution of the current density along three bars of the same phase is computed by means of the presented PEEC approach and a FEM tool. In the PEEC approach, the bars are not meshed in their length, but only in the cross section: 15 elements along the $\mathrm{z}$ axis and 4 along the $\mathrm{x}$ axis whereas in the FEM approach, air and bars are meshed with a total number of mesh elements around 600000 . So the gap between degrees of freedom number of the two methods is very different.

However, the results in Fig. 1 show that the two numerical methods are very closed, confirming that this simple PEEC meshing used to extracted parasitic resistances and inductances is efficient to model the electrical behavior of a device with only a few number of mesh elements. But to take into account the electrical couplings like the common mode loops at higher frequencies, capacitive effects have to be added to this inductive approach.

\section{PARASITIC CAPACITANCES}

\section{A. Free-Charges Computation by a Full Interaction Method}

According to the geometrical dimensions and frequency range considered, the propagation phenomena can be neglected 
$(\mathrm{L}<\lambda / 10)$. Consequently, an electrostatic formulation based on the computation of the surface distribution of the free-charges thanks to a meshing of the conductor and dielectric interfaces may be sufficient to model the capacitive effects. In order to take into account side effects and the spatial variation of surface charges, a mesh with thinner elements than the inductive ones but which is not-necessarily conformal is required. Capacitive elements will be then gathered to compute some equivalent capacitances to be connected to the R-L-M circuit.

Let us consider a surface meshing of the conductor and dielectric interfaces composed of $\mathrm{Nc}$ conductor elements and $\mathrm{Nd}$ dielectric elements. The electric potential $\mathrm{P}_{\mathrm{i}}$ at a conductor-dielectric interface and the normal field $\mathrm{E}_{\mathrm{i}}$ at a dielectric-dielectric interface (which is due to the jump of the normal field coefficient) can be written as

$$
\left\{\begin{array}{l}
\mathrm{P}_{\mathrm{i}}\left(\mathbf{r}_{\mathbf{i}}\right)=\sum_{\mathrm{j}=1}^{\mathrm{Nc}+\mathrm{Nd}} \frac{1}{\varepsilon_{0}} \iint_{\mathrm{S}_{\mathrm{j}}} \sigma_{\mathrm{j}} \cdot \mathrm{G}\left(\mathbf{r}_{\mathbf{i}}, \mathbf{r}_{\mathbf{j}}\right) \mathrm{d} \mathrm{S}_{\mathrm{j}} \\
\mathrm{E}_{\mathbf{i}}\left(\mathbf{r}_{\mathbf{i}}\right)=\sum_{\mathbf{j}=1}^{\mathrm{Nc}+\mathrm{Nd}} \frac{1}{\varepsilon_{0}} \iint_{\mathrm{S}_{\mathbf{j}}} \sigma_{\mathbf{j}} \cdot \mathrm{G}^{\prime}\left(\mathbf{r}_{\mathbf{i}}, \mathbf{r}_{\mathbf{j}}\right) \cdot \mathbf{n}_{\mathbf{j}} \mathrm{d} \mathrm{S}_{\mathbf{j}}
\end{array}\right.
$$

where $\mathbf{r}_{\mathbf{i}}$ is a point of the space, $\sigma_{\mathbf{j}}$ the surface charge of the element $\mathrm{j}, \mathrm{G}$ the green function, $\mathbf{G}^{\prime}$ the gradient of $\mathrm{G}: \mathbf{G}^{\prime}\left(\mathbf{r}, \mathbf{r}^{\prime}\right)=$ $-\left(\mathbf{r}-\mathbf{r}^{\prime}\right) /\left(4 \pi\left\|\mathbf{r}-\mathbf{r}^{\prime}\right\|^{3}\right)$ and $\mathbf{n}$, the outside normal vector. This normal field is null at the dielectric-dielectric interfaces.

An integral method, closely linked to the MoM method [7], namely the building of a matrix linear system with a full and square interaction matrix, can be solved to compute the surface charges that are assumed constant on each surface element. Traditionally with the MoM method, the coefficients of the interaction matrix are computed between each mesh center point: this is a 0-order point matching approach. This interaction matrix $\mathrm{P} / \mathrm{E}$ links the charges $\mathrm{Q}\left(\mathrm{Q}_{\mathrm{c}}\right.$ and $\mathrm{Q}_{\mathrm{d}}$ for the conductor and dielectric charges) to the potential $\mathrm{V}$ of the conductors [2-8-9]

$$
\left[\begin{array}{l}
\mathrm{P} \\
\mathrm{E}
\end{array}\right] \cdot\left[\begin{array}{l}
\mathrm{Q}_{\mathrm{c}} \\
\mathrm{Q}_{\mathrm{d}}
\end{array}\right]=\left[\begin{array}{l}
\mathrm{V} \\
0
\end{array}\right]
$$

where the coefficients are the potential coefficients $P_{i, j}$ and the normal field coefficients $E_{i, j}$ due to unit charge [9], [10]

$$
\left\{\begin{array}{l}
\mathrm{P}_{\mathrm{i}, \mathrm{j}}=1 /\left(\varepsilon_{0} \mathrm{~S}_{\mathrm{j}}\right) \iint_{\mathrm{S}_{\mathrm{j}}} \mathrm{G}\left(\mathbf{r}_{\mathbf{i}}, \mathbf{r}_{\mathbf{j}}\right) \mathrm{d} \mathrm{S}_{\mathrm{j}} \\
\mathrm{E}_{\mathrm{i}, \mathrm{j}}=\left\{\begin{array}{l}
1 /\left(\varepsilon_{0} \mathrm{~S}_{\mathrm{j}}\right) \iint_{\mathrm{S}_{\mathrm{j}}} \mathrm{G}^{\prime}\left(\mathbf{r}_{\mathbf{i}}, \mathbf{r}_{\mathbf{j}}\right) \cdot \mathbf{n}_{\mathrm{j}} \mathrm{d} \mathrm{S}_{\mathrm{j}}, \mathrm{i} \neq \mathrm{j} \\
\frac{-\varepsilon_{\mathrm{r} 1}+\varepsilon_{\mathrm{r} 2}}{2 \varepsilon_{0}\left(\varepsilon_{\mathrm{r} 1}-\varepsilon_{\mathrm{r} 2}\right) \mathrm{S}_{\mathrm{i}}} \mathrm{i}=\mathrm{j}
\end{array}\right.
\end{array}\right.
$$

$S_{j}$, the surface of the element $j$, is out the integral because the charge is assumed constant on the surface ( 0 -order). Those coefficients are respectively calculated between each Nc conductor or $\mathrm{Nd}$ dielectric elements and all $\mathrm{Nc}+\mathrm{Nd}$ elements of the meshing. The surface integrals are numerically computed thanks to the gauss point technique. The computation algorithm of the interaction matrix is totally vectorised in order to avoid double "for" loops and save time. After this computation and to improve the accuracy of the potential coefficient computation $\mathrm{P}_{\mathrm{ii}}$, analytic formulations are used [10] to correct the diagonal of the P matrix.

Other approaches with different order of integration have been developed and compared to solve the matrix system (5). In a 0-order Galerkin approach, double integrals are used to

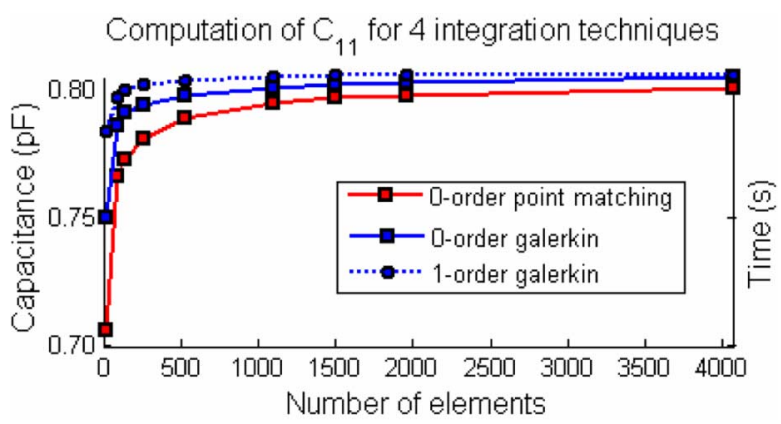

Fig. 2. Comparison of capacitance $C_{11}$ for three integration techniques depending on the number of mesh elements of two parallel plates.

compute the coefficient of (6) and the second member of the matrix linear system (6) is multiplied by the element surfaces. Potential coefficient $\mathrm{P}_{\mathbf{i}, \mathbf{i}}$ are also corrected analytically [11], [12].

The first-order integration with a triangular shape function has also been developed for a Galerkin approach. A first-order point matching method is not adapted because it would try to evaluate the potential at the nodes and the potential is singular at each node of triangular surfaces.

Fig. 2 represents the self-capacitance $\mathrm{C}_{11}$ and the integration time of the matrix P/E of one of two parallel plates $\left(10 * 10 \mathrm{~mm}^{2}\right.$, gap $2 \mathrm{~mm}$ ) for those three integration techniques. The charges used to compute these capacitances are solved by a LU-decomposition. The capacitance computation from charges will be presented at the end of this section. This figure shows that the Galerkin approach is more accurate than the point matching one. But, as far as the integration time is concerned, the 0 or first-order computation with a Galerkin method is slower than the point matching method because double integrals are computed. A nonconformal meshing can be very interesting to refine easily the areas where the gradient of potential or field are high while saving number of elements. That is why we have chosen to use 0 -order point matching.

However, the worst drawback of this integral method is the storage of the full matrix and the integration time which increases in $O\left(\mathrm{n}^{2}\right)$. To overcome this issue, the fast multipole method (FMM) appears to be very interesting to enable the modeling of large devices.

\section{B. Free-Charges Computation by the Fast Multipole Method}

At the origin, the FMM has been developed in order to accelerate the computation of far potential interactions between punctual electric charges [13]. This algorithm is low-memory consuming thanks to the use of a truncated multipole decomposition of interactions. For example the potential far from a set of $\mathrm{m}$ charges $\mathrm{Q}$ can be written in spherical coordinates as

$$
\begin{aligned}
\mathrm{V}(\rho, \theta, \varphi) & =\frac{1}{4 \pi \varepsilon_{0}} \sum_{\mathrm{n}=0}^{\infty} \sum_{\mathrm{m}=-\mathrm{n}}^{\mathrm{n}} \mathrm{M}_{\mathrm{n}}^{\mathrm{m}} \cdot \mathrm{Y}_{\mathrm{n}}^{\mathrm{m}}(\theta, \varphi) / \rho^{\mathrm{n}+1} \\
\mathrm{M}_{\mathrm{n}}^{\mathrm{m}} & =\sum_{\mathrm{i}=1}^{\mathrm{Nq}} \mathrm{Q}_{\mathrm{i}} \mathrm{r}_{\mathrm{i}}^{\mathrm{n}} \mathrm{Y}_{\mathrm{n}}^{-\mathrm{m}}\left(\alpha_{\mathrm{i}}, \beta_{\mathrm{i}}\right) \\
\mathrm{Y}_{\mathrm{n}}^{\mathrm{m}}(\theta, \varphi) & =\sqrt{\frac{(\mathrm{n}-|\mathrm{m}|) !}{(\mathrm{n}+|\mathrm{m}|) !}} \cdot \mathrm{P}_{\mathrm{n}}^{|\mathrm{m}|}(\cos \theta) \cdot \mathrm{e}^{\mathrm{i} \mathrm{m} \varphi}
\end{aligned}
$$


where $\mathrm{M}_{\mathrm{n}}^{\mathrm{m}}$, the multipole depends on the spherical harmonics $\mathrm{Y}_{\mathrm{n}}^{\mathrm{m}}$ that are composed of Legendre functions. To use these multipole series efficiently, an octree algorithm is needed to do a hierarchical partitioning of the geometry in different cube levels. This octree drives the type of interactions between each cube depending on the distance between them. To summarize the principle of the method, far interactions are computed by the FMM and the near interactions between adjacent cubes are computed by the full method presented previously.

A second version of the FMM [14], the adaptive multilevel fast multipole method (AMLFMM) is more adapted to a nonuniform distribution of charges. It allows to save memory and to accelerate even more the computation of interactions. However, in our problem, charges are not punctual but linked to surface and the meshing can be nonconform, but the second part of the FMM theorems ensures an upper bound of the error of the potential, computed by a multipole decomposition, due to a punctual distribution of charges. This maximum error value is not guaranteed with surface charges because the cube partitioning done by the octree algorithm does not deal with these surfaces. Consequently, we have developed a new version, quite similar to [15], that adaptively takes into account the size of the surface mesh element in the octree in order to better evaluate the proximity of the mesh elements and their belonging to a cube at a certain level.

Concerning the far electric field with the FMM, the computational effort, namely the differentiation of the electric potential $(\mathrm{E}=-\nabla \mathrm{V})$, is relatively weak because only the last coefficient $\mathrm{Y}_{\mathrm{n}}^{\mathrm{m}}(\theta, \varphi) / \rho^{\mathrm{n}+1}$ in (8) depends on the evaluation point coordinates. Consequently, all the multipole coefficients used to evaluate the electric potential are also used to compute the electric field. This last one converted into Cartesian coordinates by means of a jacobian matrix is multiplied by the unit normal vector of surface.

To solve the problem with the FMM, an iterative solver is necessarily used. Because there is no "true" interaction matrix, a left preconditioned GMRESR(m) algorithm [16] is chosen. Thanks to the near integration, all the full and square small matrixes are inverted and used in the preconditioning [17]. Comparisons of integration and resolution time on the two plates example, plotted in the Fig. 3, shows the dramatic rapidity of the FMM algorithm developed. Moreover, the memory consumption is very low: in the full method, to integer and solve the two plates meshed in 10000 elements, $3 \mathrm{~Gb}$ of memory are needed (because of the storage of the full and square interaction matrix of size $10000^{2}$ ) whereas with the FMM algorithm, a problem of 300000 elements requires less than $1.6 \mathrm{~Gb}$.

\section{Computation of Capacitances From Free-Charges}

Let us consider $\mathrm{N}$ conductors, a $\mathrm{N}^{2}$ square capacitance matrix between the conductors is computed and models the main capacitive electric couplings between the $\mathrm{N}$ conductors of the device (or part of conductors). The capacitance coefficient $\mathrm{C}_{\mathrm{i}, \mathrm{j}}$ is computed by adding charge sets that are solved from the full matrix system (5) presented above or with the FMM

$$
\begin{aligned}
\mathrm{C}_{\mathrm{i}, \mathrm{j}}=\sum_{\mathrm{k}=1}^{\mathrm{Nc}(\mathrm{j})} \varepsilon_{\mathrm{rk}}^{(\mathrm{j})} \mathrm{Q}_{\mathrm{ck}}^{(\mathrm{j})} \operatorname{Cond}(\mathrm{i}) & =1 \mathrm{~V} \\
\operatorname{Cond}(\mathrm{j}) & =0 \mathrm{~V}, \mathrm{i} \neq \mathrm{j}
\end{aligned} .
$$

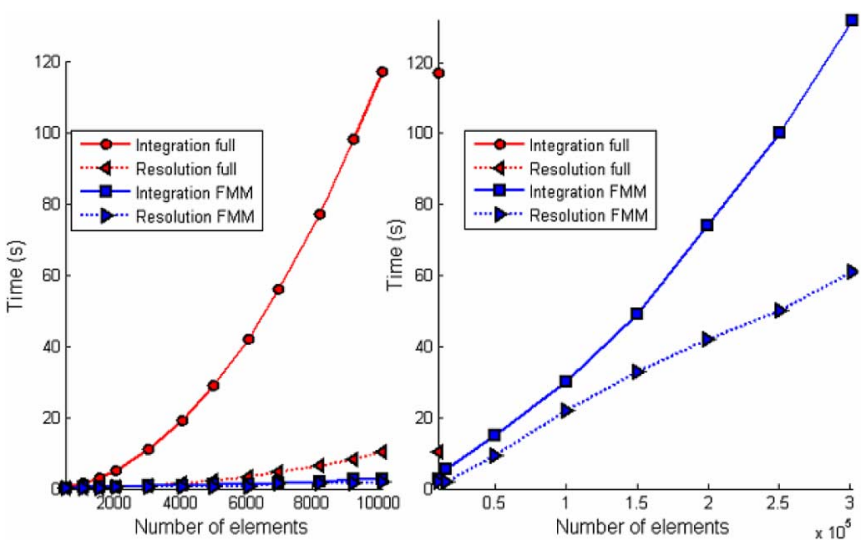

Fig. 3. Comparison integration and resolution time (GMRES) between a full method and a FMM.

The $\mathrm{Nc}(\mathrm{j})$ charges $\mathrm{Q}_{\mathrm{ck}}^{(\mathrm{j})}$ are located on the conductor $\mathrm{j}$ and are obtained by performing the linear system with the following conditions: the potential of the conductor $i$ is set to $1 \mathrm{~V}$ and $0 \mathrm{~V}$ for the others. The term $\varepsilon_{\mathrm{rk}}^{(j)}$ takes into account the nature of the interface with a dielectric medium and applies 1 , if it is air and $\varepsilon_{\mathrm{r}}$ (or $\left(\varepsilon_{\mathrm{r}}+1\right) / 2$ [18]), if it is a dielectric material and the conductor is a volume (or a surface). The capacitances coefficient $\mathrm{C}_{\mathrm{ii}}$ and $\mathrm{C}_{\mathrm{ij}}$ are respectively positive and negative, but we do not directly use this matrix in the lumped equivalent circuit. We prefer to use the more practical Kirchhoff's definition for the capacitances, i.e., so that, the potential of conductors are referenced to the ground as

$$
\mathrm{Q}_{\mathrm{ci}}=\mathrm{C}_{\mathrm{i}, \mathrm{i}}^{\prime} \mathrm{V}_{\mathrm{i}}+\sum_{\mathrm{j}=1}^{\mathrm{N}} \mathrm{C}_{\mathrm{i}, \mathrm{j}}^{\prime}\left(\mathrm{V}_{\mathrm{i}}-\mathrm{V}_{\mathrm{j}}\right)
$$

with

$$
\left\{\begin{array}{l}
\mathrm{C}_{\mathrm{i}, \mathrm{i}}^{\prime}=\sum_{\mathrm{j}=1}^{\mathrm{N}} \mathrm{C}_{\mathrm{i}, \mathrm{j}} \\
\mathrm{C}_{\mathrm{i}, \mathrm{j}}=-\mathrm{C}_{\mathrm{i}, \mathrm{j}} \quad \text { if } \mathrm{i} \neq \mathrm{j}
\end{array}\right.
$$

where $\mathrm{Q}_{\mathrm{ci}}$ is the total charge of the conductor $\mathrm{i}$ of potential $\mathrm{V}_{\mathrm{i}}$ referenced to the ground $\left(\mathrm{V}_{\text {ground }}=0 \mathrm{~V}\right)$. A well-known and simple relation (11) permits to obtain the usable capacitance matrix $C^{\prime}$ from the matrix $C$ [19].

\section{Construction of the Complete (R-L-M-C) PARAsitic EQUIVALENT CIRCUIT}

The inductive and capacitive parasitic parameters are not directly connected. First the inductive problem is reduced as regards the number of degrees of freedom (Fig. 4). For example, the inductive RLM matrix between the pins $\mathrm{A}_{1}, \mathrm{~A}_{2}, \mathrm{~B}_{1}$ and $\mathrm{B}_{2}$ of the Fig. 4 is reduced in a matrix $2 * 2$. In the circuit simulator, currents are not solved in each inductive element because it would require too much memory and solving-time at each time step. Let us consider $\mathrm{U}_{\mathrm{b}}, \mathrm{I}_{\mathrm{b}}, \mathrm{Z}_{\mathrm{b}}$ and $\mathrm{U}_{\mathrm{s}}$ respectively the branch voltage and branch current, the impedance matrix composed of the last presented parasitic RLM matrix and eventually other R, L, or C passive elements, and to finish the source voltage. Thus, the full electric system can be written as

$$
\mathrm{U}_{\mathrm{b}}=\mathrm{Z}_{\mathrm{b}} \mathrm{I}_{\mathrm{b}}+\mathrm{U}_{\mathrm{s}} \text {. }
$$




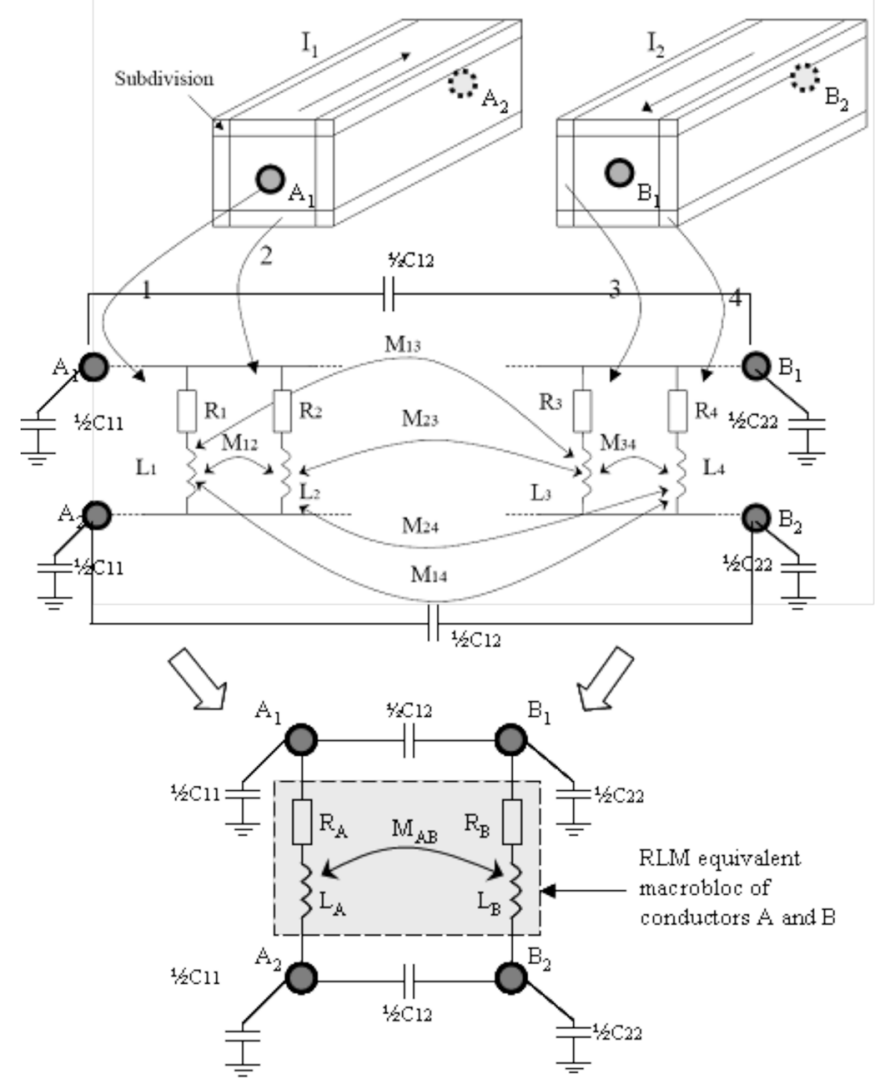

Fig. 4. Construction of the complete parasitic equivalent circuit of two unidirectional conductors.

Actually, a reduced equivalent inductive matrix $Z_{r}$ is extracted between a set of chosen electric nodes. It is computed at a chosen frequency. Among these nodes, there are those used to connect the parasitic capacitances, the power supply, the load and other electric components of power or command. To reduce the system (11) a matrix M of independent Kirchhoff's loops is used. $\mathrm{M}$ is composed of 0,1 or -1 and describes the connectivity of each inductive element [20]. It is possible to choose $\mathrm{M}$ as $\mathrm{M} \cdot \mathrm{U}_{\mathrm{b}}=0$. Thus, we can have a set of currents in the independent branches as $I_{m}=M^{T} \cdot I_{b}$. Thus, by multiplying (11) at the left by $\mathrm{M}$ and with $\mathrm{Z}_{\mathrm{m}}=\mathrm{M} \cdot \mathrm{Z}_{\mathrm{b}} \cdot \mathrm{M}^{\mathrm{T}}$, we can write

$$
\mathrm{Z}_{\mathrm{m}} \cdot \mathrm{I}_{\mathrm{m}}+\mathrm{M} \cdot \mathrm{U}_{\mathrm{s}}=0 .
$$

After a transformation of $\mathrm{Z}_{\mathrm{m}}$ into a partial upper triangular matrix with a Gaussian elimination process, we can obtain the small impedance matrix $Z_{r}$ composed of equivalent impedances between the chosen electric nodes

$$
\mathrm{Z}_{\mathrm{m}}=\left[\begin{array}{cccc}
* & \cdots & \cdots & * \\
0 & \ddots & & \vdots \\
\vdots & \ddots & * & * \\
0 & \cdots & 0 & \mathrm{Z}_{\mathrm{r}}
\end{array}\right] .
$$

All the other impedances between other nodes, noted ${ }^{*}$, are not used. From $Z_{r}$, a macroblock is built with the same number of pins that the size of $Z_{r}$ and it represents the equivalent impedance at the chosen frequency.
The parasitic capacitances are directly connected to this macroblock. The self capacitive effect of each unidirectional conductor is divided in the two extremities and connected to a reference ground. The coupling capacitive effects with other unidirectional conductors are also divided into their extremities (Fig. 4). To increase the capacitive couplings of very long conductors, it is possible to split those in $\mathrm{k}$ subdivisions and to proceed in the same way for the $\mathrm{k}$ parts. Thus, capacitive and inductive couplings are modeled by $\Pi$ cells which are more convenient to connect than $\mathrm{T}$ cells as extremity nodes of conductors are accessible via the macroblock pins (and not the middle node).

The capacitances between bidirectional conductors (ground planes) and unidirectional conductors are connected in several particular points to accurately take into account proximity effects. Thus, a complete equivalent circuit of interconnections is built. By connecting the rest of the electrical circuit (power, command, etc.) it is possible to simulate the electrical behavior of the power device in a circuit simulator.

\section{Application to an Industrial Variable SpeEd Drive}

\section{A. Extraction of the (R-L-M-C) Parasitic Equivalent Circuit}

These methods presented previously have been used to model a complex real static converter: a marketed variable speed drive.

It is composed of a common mode filter, a power and command module, a mechanical and cabling part. Its function is to drive a three-phase motor depending on a command signal order. The power module is composed of four thin copper-track layers. To analyse this complex device, a system-coupling approach is used.

All of this inductive method described is implemented in InCa3D, a commercially available software [21]. From the inductive matrix, postprocessing modeling gives an accurate description of the current density distribution and losses in the conductors or near magnetic field [2]. A coupling with a circuit solver, a SPICE-like tool, makes possible time-domain analysis by using a reduced R-L-M matrix, the macroblock mentioned before.

The Fig. 5 shows the 1-D (tracks) and 2-D (mechanical parts or ground planes) meshings used to extract the lumped elements: a square resistive-inductive matrix $(6800 * 6800$ elements). The capacitive meshing contains 48500 surface elements. 27 conductors are identified and the capacitive matrix is computed with the FMM algorithm and the GMRESR $(\mathrm{m})$ solver. To finish, a system-level software, Saber [22], is used to build the complete PEEC circuit of the interconnections of this device made of three R-L-M macroblocks and all the capacitances which are linked themselves (Fig. 6).

\section{B. Time-Domain Simulations}

The supply chain, the load (a three-phase motor) and the command circuit are then added to the previous PEEC circuit. The output DC bus voltage is of $538 \mathrm{~V}$. It is close to the theory (548 $\mathrm{V}$, i.e., gap of $2 \%$ ). The PEEC circuit has a non-negligible influence on the inverter voltages especially during the commutations of the complementary inverter arms because these last ones have higher frequency signals (Fig. 6). The equivalent circuit of such industrial device is complex. In consequence, it has been verified that the model is in agreements with the impedance of 


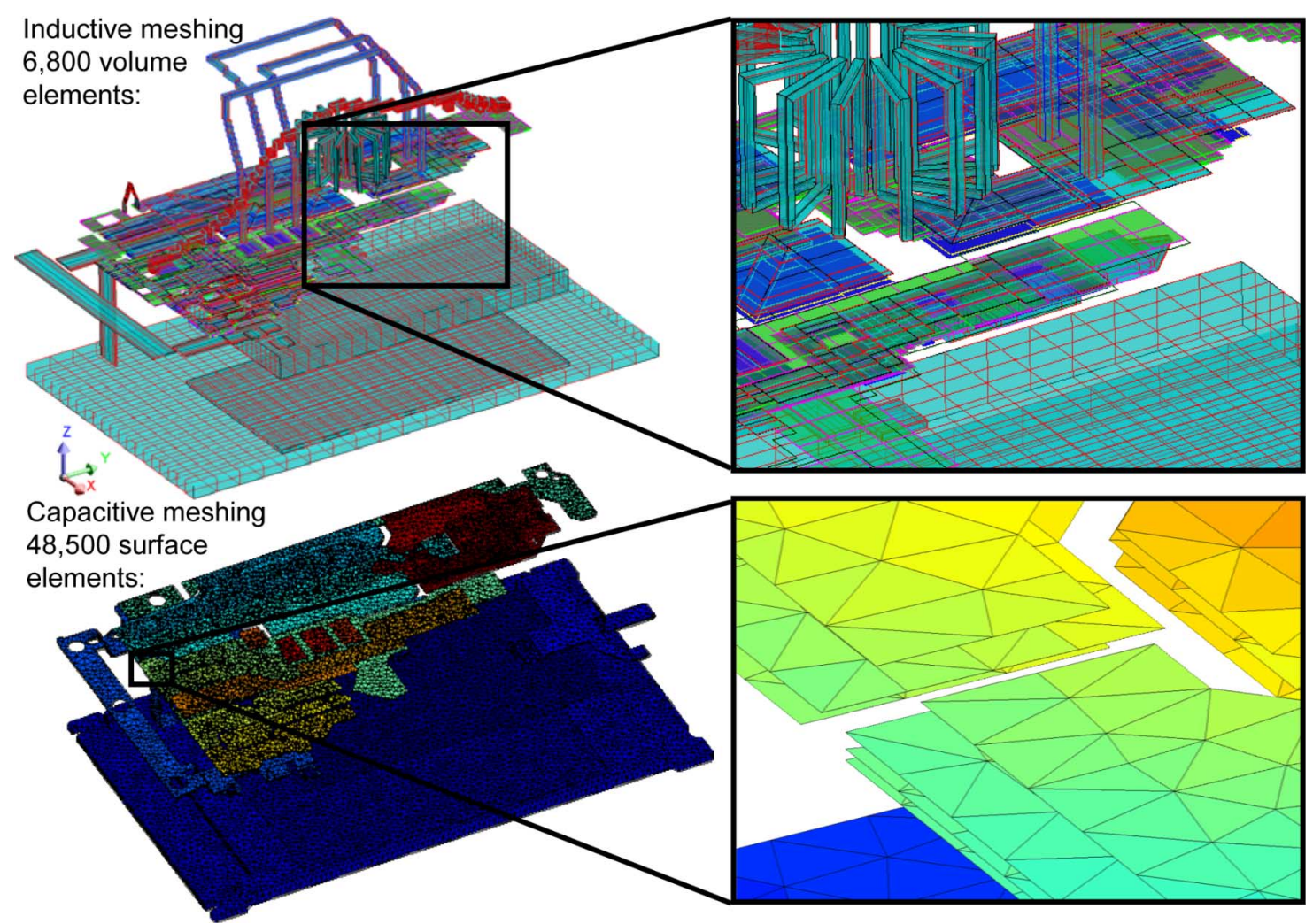

Fig. 5. Inductive and capacitive meshing of the industrial variable speed drive.

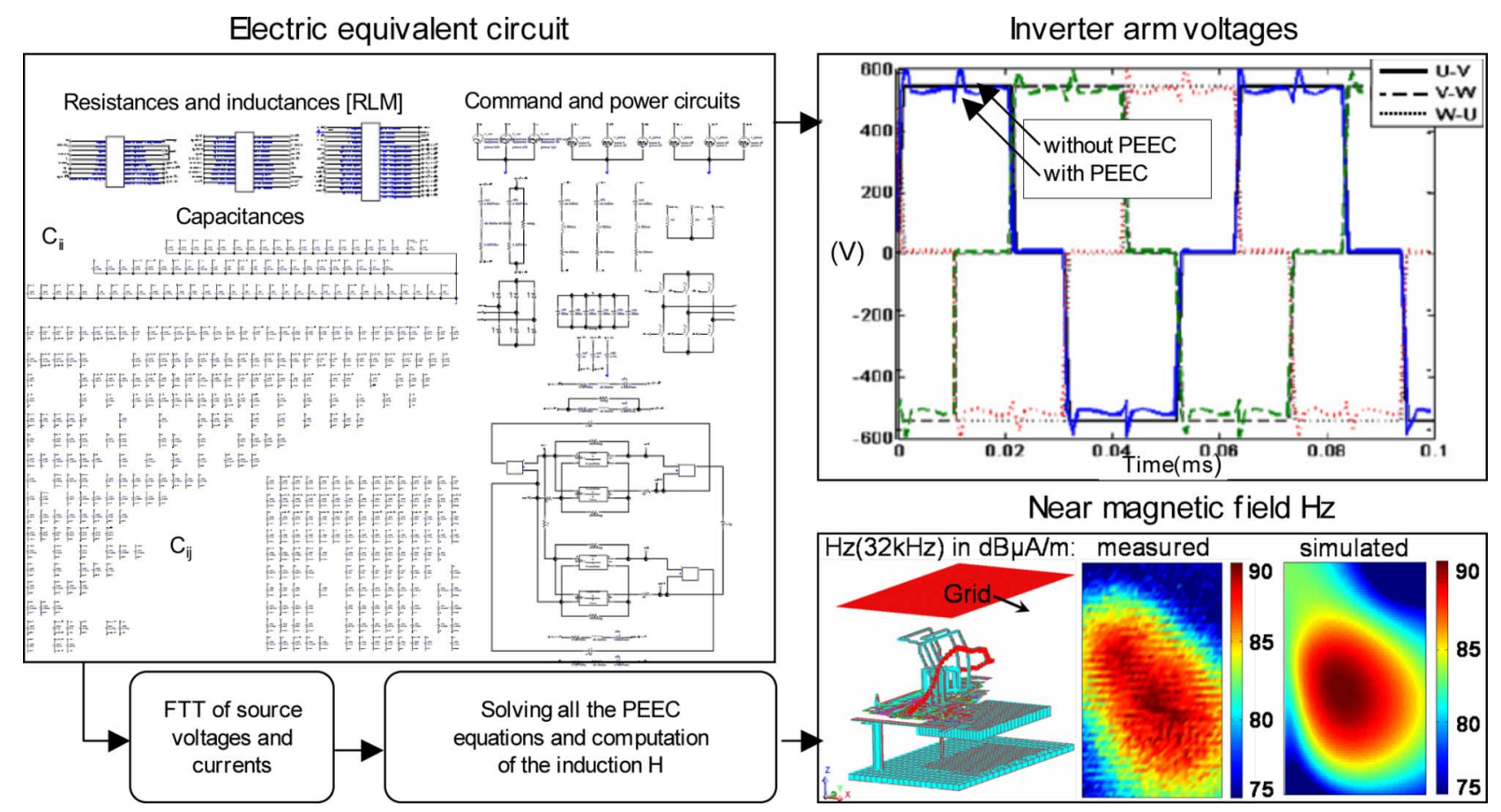

Fig. 6. Modeling chain of the industrial variable speed drive.

the variable speed drive. To do that, the resonances in the conducted frequency range $(150 \mathrm{kHz}-30 \mathrm{MHz})$ are measured and modeled (Table I). It can be seen that the simulated and measured resonances are close. The F2 resonance which is linked to the cable connecting the converter to the motor does not appear because it is not modeled in the simulation circuit.
TABLE I

MEASURED AND Simulated RESONANCE FREQUENCIES

\begin{tabular}{|l|c|c|c|c|}
\hline Frequencies in $\mathrm{MHz}$ & $\mathrm{F} 0$ & $\mathrm{~F} 1$ & $\mathrm{~F} 2$ & $\mathrm{~F} 3$ \\
\hline Measured & 1.314 & 5.166 & 19.16 & 25.83 \\
\hline Simulated & 1.221 & 5.198 & none & 24.76 \\
\hline
\end{tabular}




\section{Near Magnetic Field Studies}

Then, a second analysis of the emitted near magnetic field, just over the static converter, is achieved. The Fourier transform of source currents and voltages, which are obtained by a time-domain simulation, are introduced as sources in our model. Then current distribution is solved and the magnetic field emitted is computed at a given frequency by means of the Biot and Savart law: the magnetic field $\mathbf{B}$ created by the $\mathrm{NbVol}$ inductive volume mesh elements of current density $\mathbf{j}$ is $(\mathbf{H}=\mu \mathbf{B})$

$$
\mathbf{B}(\mathbf{r})=\mu_{0} \sum_{\mathrm{i}=1}^{\mathrm{NbVol}} \iint_{\mathrm{V}_{\mathbf{i}}} \int \mathbf{G}^{\prime}\left(\mathbf{r}, \mathbf{r}_{\mathbf{i}}\right) \cdot \mathbf{j}_{\mathbf{i}} \mathrm{dV} \mathrm{V}_{\mathbf{i}} .
$$

The comparison between the vertical coordinate of the magnetic field $\mathrm{Hz}$ at $32-\mathrm{kHz}$ simulated and measured is presented in Fig. 6. A good agreement between the two shades can be appreciated. It means that the main current loops are correctly modeled. These good results validate the methodology of all the modeling chain used to model this industrial device.

\section{CONCLUSION}

In this paper, the coupling between two adapted integral methods has been presented. From two adapted meshings allowing, respectively, resistive-inductive and capacitive equivalent elements of power interconnections of industrial complexity have been extracted. The use of an adapted FMM to compute parasitic capacitances and the reduction technique of the RLM matrix into a smaller macroblock permits to deal with large and complex geometries with relatively few memory consumption.

It has also been highlighted that the complete (R-L-M-C) equivalent circuit can be exported into a SPICE-like tool where time-domain analysis can be performed. Then, the Fourier transforms of the currents and voltages obtained have been introduced as sources in the 3-D geometric representation of the interconnections in order to analyze the EMC performances of the system on a wide frequency range. The electrical behavior, the harmonic response and the emitted near magnetic field simulated compared to those obtained with measurements have validated this system-coupling approach on a real industrial variable speed drive.

\section{ACKNOWLEDGMENT}

The authors would like to thank J. Ecrabey from Schneider Electric and P. Loizelet from STIE for lending the variable speed drive and also O. Aouine and C. Labarre from ENSM for the near magnetic field measurement. In addition, the authors acknowledge P. Labie for the helpful programming around the FMM.

\section{REFERENCES}

[1] A. E. Ruehli and A. C. Cangellaris, "Progress in the methodologies for the electrical modeling of interconnects and electronic packages," Proc. IEEE, vol. 89, no. 5, May 2001.

[2] V. Ardon, J. Aimé, O. Chadebec, E. Clavel, and E. Vialardi, "MoM and PEEC method to reach a complete equivalent circuit of a static converter," presented at the EMC Zurich, Zurich, Switzerland, Jan. 2009.

[3] E. Clavel, J. Roudet, and J.-L. Schanen, "A multiconductor transmission line method to study non-perfect ground planes," in Proc. IEEE EMC, Zurich, Switzerland, 1999, pp. 437-440.

[4] A. E. Ruehli, "Survey of computer-aided electrical analysis of integrated circuit interconnections," IBM J. Res. Develop., vol. 23, pp. 626-639, Nov. 1979.

[5] J. Aimé, J. Roudet, E. Clavel, O. Aouine, C. Labarre, F. Costa, and J. Ecrabey, "Prediction and measurement of the magnetic near field of a static converter," presented at the IEEE Int. Res. Symp. Industrial Electronics, Jun. 2007.

[6] J.-M. Guichon, "Modélisation, caractérisation, dimensionnement de jeux de barres," Ph.D. dissertation of INPG, Grenoble, France, 2001.

[7] O. Chadebec, J.-L. Coulomb, and F. Janet, "A review of magnetostatic moment method," IEEE Trans. Magn., vol. 42, no. 4, pp. 515-520, Apr. 2005.

[8] K. Nabors and J. White, "Multipole-accelerated capacitance for 3-D structures with multiple dielectrics," IEEE Trans. Circuits Syst., vol. 39, no. 11, Nov. 1992.

[9] V. Ardon, J. Aimé, E. Clavel, O. Chadebec, E. Vialardi, J.-M. Guichon, and P. Labie, "EMC analysis of static converters by the extraction of a complete equivalent circuit via a dedicated PEEC method," presented at the 8th Int. Symp. Electric and Magnetic Fields, May 2009.

[10] "Tome I-Les distributions," Electrostatique, chp—Distributions superficielles p. 246, Durand, Masson.

[11] P. Arcioni, M. Bressan, and L. Perregrini, "On the evaluation of the double surface integrals arising in the application of the boundary integral method to 3-d problems," IEEE Trans. Microw. Theory Tech., vol. 45, no. 3, pp. 436-439, 1997.

[12] D. R. Wilton, S. M. Rao, A. W. Glisson, D. H. Schaubert, O. M. Al-Bundak, and C. M. Butler, "Potential integrals for uniform and linear source distributions on polygonal and polyhedral domains," IEEE Trans. Antennas Propagat., vol. AP-32, pp. 276-281, Mar. 1984.

[13] L. Greengard and V. Rokhlin, "A fast algorithm for particle simulations," J. Comput. Phys., vol. 73, pp. 325-348, 1987.

[14] J. Carrier, L. Greengard, and V. Rokhlin, "A fast adaptive multipole algorithm for particle simulations," SIAM J. Sci. Statist. Comput., 1988.

[15] V. Jandhyala, S. Savage, E. Bracken, and Z. Cendes, "Efficient capacitance computation for structures with non-uniform adaptive surface meshes," presented at the ACM/IEEE Design Automation Conf., 1999.

[16] Y. Saad and M. H. Schultz, "GMRES: A generalized minimal residual algorithm for solving nonsymmetric linear systems," SIAM J. Sci. Statist. Comput., vol. 7, pp. 856-869, Jul. 1986.

[17] K. Nabors, F. Korsmeyer, F. Leighton, and J. White, "Preconditioned, adaptive, multipole-accelerated iterative methods for three-dimensional first-kind integral equations of potential theory," SIAM J. Sci. Comput., vol. 15, no. 3, pp. 713-735, May 1994.

[18] C. R. Paul, Introduction to Electromagnetic Compatibility. Hoboken, NJ: Wiley, 1992.

[19] C. R. Paul, "Useful matrix chain parameter identities for the analysis of multiconductor transmission lines," IEEE Trans. Microw. Theory Tech., vol. 23, no. 9, pp. 756-760, Sep. 1975.

[20] A. Desoer and E. S. Kuh, Basic Circuit Theory. New York: McGrawHill, 1969.

[21] [Online]. Available: http://www.cedrat.com, InCa3D software, Cedrat

[22] [Online]. Available: http://www.avanticorp.com, Saber software, Avanti 